\title{
Les nouvelles figures du travailleur immigré : fragmentation des statuts d'emploi et européanisation des migrations
}

New Figures of Migrant Workers: Fragmentation of Work Status and Europeanization of Migration

Nuevas figuras de trabajadores migrantes: fragmentación del estatus laboral y la europeización de la migración

\section{Andrea Rea}

\section{OpenEdition}

\section{Journals}

Édition électronique

URL : https://journals.openedition.org/remi/6371

DOI : 10.4000/remi.6371

ISSN : $1777-5418$

Éditeur

Université de Poitiers

Édition imprimée

Date de publication : 1 juin 2013

Pagination : 15-35

ISBN : 979-10-90426-08-5

ISSN : 0765-0752

Référence électronique

Andrea Rea, «Les nouvelles figures du travailleur immigré : fragmentation des statuts d'emploi et européanisation des migrations », Revue européenne des migrations internationales [En ligne], vol. 29 $n^{\circ} 2$ | 2013, mis en ligne le 01 juin 2016, consulté le 15 avril 2022. URL : http://journals.openedition.org/ remi/6371; DOI : https://doi.org/10.4000/remi.6371 


\title{
Les nouvelles figures du travailleur immigré : fragmentation des statuts d'emploi et européanisation des migrations
}

\author{
Andrea Rea ${ }^{1}$
}

\section{Introduction}

La Belgique est un pays d'immigration depuis la fin de la Deuxième Guerre mondiale et le reste malgré une politique restrictive mise en place depuis plusieurs années. Au cours des vingt dernières années, le nombre d'étrangers n'a pas cessé d'augmenter, phénomène lié notamment au paradoxe libéral formulé par James Hollifield (1997) rappelant que les forces économiques poussent à l'ouverture des frontières alors que les forces politiques internes soutiennent plutôt la fermeture. La Belgique partage avec la plupart des pays du nord de l'Europe (France, Allemagne, Pays-Bas) un même modèle de politique migratoire comme le précise Freeman (1995). Les nouvelles migrations en Belgique résultent principalement de l'application de droits attribués aux étrangers, en particulier le droit au regroupement familial et la politique d'asile et d'aide humanitaire. L'immigration de travail est relativement réduite. Selon les statistiques d'attribution des titres de séjour, ceux délivrés pour des raisons de travail ne représentent que 6,3\% de tous les titres de séjour attribués en 2010. Ceci laisserait penser que les nouvelles migrations de travail sont marginales en Belgique. Cette donnée statistique occulte une part importante des nouvelles modalités de l'immigration de travail. Cette contribution vise à montrer que la migration de travail est néanmoins plus importante que ne le laissent penser les données relatives aux permis de travail, instruments traditionnels de la politique migratoire de travail. Le travail immigré se compose dorénavant de multiples figures de travailleurs migrants (Rea, 2010). Trois processus interdépendants affectent les nouvelles modalités contemporaines du travail immigré. Le premier, le plus connu, concerne l'accroissement du travail informel et en particulier celui des sans-papiers (Adam et al., 2002 ; Martiniello et al., 2010). Le deuxième tient au recrutement important de travailleurs provenant des nouveaux États membres de I'UE, et tout particulièrement les Polonais, les Roumains et 
les Bulgares. Enfin, le troisième concerne les transformations des modes de recrutement des travailleurs en lien avec la segmentation des statuts du travail. Cette contribution entend dépasser certaines catégorisations juridiques qui limitent la compréhension de l'usage des travailleurs étrangers sur le marché du travail. Ainsi, nous souhaitons proposer une analyse de l'immigration de travail dépassant certaines frontières normatives et cognitives. II s'agit avant tout de prendre en considération comme migration de travail les postes octroyés à des ressortissants des nouveaux États membres de I'UE (27). II convient de sortir de l'opposition parfois trop radicale et étanche entre migrations européennes (UE) et migrations non-européennes, qualifiant les premières de mobilité et les secondes de migration par les textes européens. Les deux sont des migrations de travail nécessitant une analyse conjointe. Nous allons montrer qu'il existe un continuum entre ces deux types de migration de travail qui repose sur la prise en compte dans l'imbrication sur les lieux de travail des multiples statuts possibles (salariés avec permis de travail, indépendants, travailleurs détachés). Cette contribution se concentre uniquement sur les nouvelles migrations de travail et ne traite pas d'une autre problématique importante, à savoir, celle de l'insertion professionnelle des Belges d'origine étrangère particulièrement affectés par le chômage et la discrimination raciale à l'embauche².

\section{Articulation des origines nationales et des statuts sur le marché du travail}

En regard de la période fordiste, l'immigration de travail s'est fortement diversifiée, notamment en raison des changements de statuts sur le marché du travail, de leur précarisation et de leur fragmentation. Le travail immigré peut prendre diverses formes qui articulent statut à l'emploi et statut d'étranger : travail saisonnier, travail sans obligation de permis de travail (les ressortissants UE et les immigrés ayant un permis de séjour permanent), travail régulé par des permis de travail (les Européens soumis à des mesures transitoires et les étrangers non UE), statut d'indépendant (vrai ou faux), le travail des étudiants étrangers, le travail des demandeurs d'asile, le travail irrégulier, le détachement de travailleurs. Ces changements se sont opérés alors même que la philosophie générale de l'usage du travail migrant reste plus que jamais celle du début des politiques migratoires en Europe : une migration temporaire. Les textes européens (EMN, 2010) en la matière ne laissent aucun doute sur la priorité octroyée à l'immigration temporaire ou circulaire. Les migrations de travail n'ont pas pour vocation l'établissement des immigrés; ils restent des oiseaux de passage selon l'expression utilisée par Michael J. Piore (1979). Comme par le passé, les nouveaux travailleurs migrants sont confrontés à une forte segmentation du marché du travail qui les voit surreprésentés dans certains segments.

Pour Stephen Castles (2002), la mondialisation conduit plus que jamais à faire appel à l'immigration pour les " 3-D Jobs " (Dirty, Demanding and Dangerous). Cependant, l'occupation de la main-d'œuvre étrangère a connu un déplacement

2 Je tiens particulièrement à remercier Marie Godin, Martin Rosenfeld, Elzbieta Kuzma et Beatriz Camargo, chercheurs du GERME dont certains résultats de leurs recherches sont repris dans ce papier. 
des secteurs d'activités de l'industrie vers les services, expliquant par ailleurs l'explosion de la féminisation de la migration de travail (Catarino et Morokvasic, 2005 ; Timmerman et al., 2012). En Belgique, comme partout en Europe, I'horticulture, la construction, I'hôtellerie, la restauration, la confection et les services représentent des secteurs d'activité à haute intensité de travailleurs immigrés. Schématiquement deux périodes peuvent être distinguées. La première est celle des années 1990 au cours desquelles le travail immigré prend majoritairement la forme du travail irrégulier assuré par les sans-papiers (Adam et al., 2002). Cette catégorie se compose principalement de demandeurs d'asile dont la procédure est extrêmement longue, souvent plus de huit ans à l'époque, et qui ne trouvent à survivre qu'en travaillant au noir. Parmi les sans-papiers figurent aussi ceux que l'on nomme les overstayers, à savoir les étrangers qui sont entrés légalement mais qui restent sur le territoire après l'expiration de leur titre de séjour ou d'entrée sur le territoire (étudiants, travailleurs temporaires, touristes) auxquels s'ajoutent les étrangers irréguliers qui ne sont jamais entrés légalement sur le territoire. Durant ces années arrivent aussi de nombreux migrants polonais qui n'ont plus besoin depuis 1991 d'un visa touristique pour entrer sur le territoire. Ces sans-papiers vivaient à l'intérieur des frontières de l'État tout en étant maintenus aux frontières des institutions de l'État de droit et de l'État social. La clandestinité a été, et reste encore, pour certains, un statut social durable. Comme le montrent Alain Morice (2006) et d'autres, le maintien des travailleurs immigrés dans un statut d'irréguliers, par rapport à leur séjour ou par rapport à leur statut dans l'emploi, contribue à accroître leur assujettissement à leurs employeurs, et par conséquent, à réduire leur liberté et leur mobilité. Le travail des immigrés sans-papiers se concentre alors dans des segments du marché du travail peu délocalisables (horticulture, restaurant, construction, etc.) et où les travailleurs étrangers sont principalement des travailleurs irréguliers faisant l'objet de contrôles accrus de la part de l'inspection sociale. Ce processus a été qualifié de délocalisation sur place par Emmanuel Terray (1999). Durant les années 2000, un autre processus important prend place, s'ajoutant au précédent. Dans le cadre de l'élargissement de I'UE, on a assisté à un double mouvement. Le premier consiste en une délocalisation des entreprises, surtout industrielles, de I'UE-15 vers les douze nouveaux États membres. Ces délocalisations servent à l'accroissement de la marge bénéficiaire des entreprises en produisant là où la main-d'œuvre est moins coûteuse. Ce processus est accompagné d'un autre que I'on peut qualifier de délocalisations intérieures. De nombreux pays européens du Nord et du Sud vont chercher les travailleurs immigrés parmi les ressortissants des nouveaux États membres de I'UE. II y a ainsi une internalisation au sein de l'espace européen du bassin de recrutement d'une partie des travailleurs migrants. Comme durant l'entre-deux-guerres, la Pologne redevient un pourvoyeur de main-d'œuvre important pour des pays comme l'Allemagne, les Pays-Bas, la Belgique, le Royaume-Uni mais aussi I'Espagne et I'Italie.

Le recours au travail irrégulier des sans-papiers et au travail régulier de travailleurs immigrés de pays de l'Europe centrale et orientale est également affecté par les changements des statuts sur le marché du travail. Là où existait une massification statutaire du salarié de l'époque fordiste, à laquelle a participé le modèle du Gastarbeiter, nous trouvons à présent une fragmentation statutaire des contrats d'emploi (Supiot, 1999). Ce morcellement résulte d'une part, de la multiplication des régimes de contrat de travail, et d'autre part, de l'émiettement des activités entrepreneuriales, notamment en raison du recours à la sous-trai- 
tance en cascade ou au détachement de personnel, ces deux derniers processus s'articulant. Ils s'inscrivent dans les pratiques relevant de la liberté de circulation des services. Des entreprises ayant leur siège social dans un autre pays européen que la Belgique viennent y exécuter un contrat de services avec leurs travailleurs sans que ceux-ci ne soient soumis à la réglementation belge sur le travail immigré. Les travailleurs détachés sont attachés à leur entreprise. S'ils perçoivent un salaire selon les normes belges, la protection sociale est garantie selon la législation du pays du siège social de l'entreprise. Ceci permet de recourir à une "immigration sans travailleurs immigrés" (Rea, 2006). En effet, ce type d'allocation de main-d'œuvre ouvre une nouvelle manière de penser l'immigration : ces travailleurs ne sont plus liés à l'État dans lequel ils travaillent temporairement, mais à l'entreprise qui les embauche. L'État perd une partie de son contrôle sur ces travailleurs. Comme pour la délocalisation des entreprises, le détachement de travailleurs en tant que délocalisation intérieure relève d'une pratique de dumping social. En l'absence de contrôle systématique, il n'est pas impossible, en outre, que ces entreprises recrutent également des travailleurs étrangers irréguliers dans le pays où elles ont leur siège social.

Enfin, la transformation des entreprises fordistes, leur découpage, les processus d'externalisation qui ont conduit à réduire leur taille et à changer les statuts des travailleurs produisent également une fragmentation des migrations de travail à l'image de la fragmentation des entreprises. De nouvelles réglementations en matière $d^{\prime}$ 'usage de sous-traitance accroissent la précarité des conditions de travail liée notamment à l'externalisation d'activités vers des petites entreprises, et peuvent également favoriser le recours à du travail irrégulier. Pour être compétitives sur le marché international, des "zones de travail gris " se constituent où des travailleurs immigrés déclarés côtoient des irréguliers (Brun, 2003). Enfin, la complexification des migrations de travail repose également sur l'extension de la fonction d'entreprise à ce que les économistes classiques nomment les ménages. Dans le domaine de la domesticité, de l'aide aux personnes, du soutien à l'éducation des enfants, les acteurs économiques ne sont souvent pas des entreprises mais des familles, des personnes privées. Ce processus conduit à une sorte de privatisation des fonctions de l'État social (Lutz, 2008), en particulier là où il est moins développé, par substitution des institutions étatiques par des travailleuses immigrées de plus en plus soumises à un modèle d'intégration subalterne, comme le qualifie Maurizio Ambrosini (2005). La massification de l'usage des femmes immigrées pour régler les questions de la sphère privée (conciliation de la vie professionnelle et privée, entraide familiale) des familles autochtones confirme la thèse de Bridget Anderson (2000) affirmant que le ménage privé constitue un marché mondialisé de la maind'œuvre féminine.

Pour la Belgique, l'élargissement de I'UE a été une opportunité pour réorienter son recrutement de la main-d'œuvre étrangère. II est possible de dire qu'aujourd'hui en Belgique une majorité des travailleurs immigrés, en particulier temporaires, ne sont pas des ressortissants des pays tiers. Les travailleurs immigrés non-européens se comptent surtout parmi les étrangers bénéficiaires de l'une des deux campagnes de régularisation en Belgique en 2000 (+/40 000 personnes) et en 2010 (+/34 000 personnes). Ayant obtenu un titre de séjour permanent pour la plupart d'entre eux, ils ne sont pas soumis à l'obligation d'avoir un permis de travail. Une autre partie des travailleurs immigrés non 
européens entrent sur le marché du travail de manière dérivée parce qu'ils sont arrivés d'abord dans le cadre du regroupement familial. Enfin, les travailleurs immigrés des pays tiers disposant d'un permis de travail se trouvent surtout parmi la catégorie des travailleurs hautement qualifiés.

Entre 2004 et 2010, le travail immigré en tant que facteur d'adaptation conjoncturelle du marché du travail concerne prioritairement les Polonais, les Roumains et les Bulgares. On retrouve en cela un ancien mécanisme associé à ce que I'on qualifie dorénavant de "préférence communautaire ", à savoir que I'UE se développe en internalisant un bassin de recrutement de main-d'œuvre bon marché. Ce fut le cas pour les Italiens dans I'Europe des 6, ce fut partiellement le cas avec l'Espagne, le Portugal et la Grèce dans l'Europe des 12. Par la suite, les mécanismes d'homogénéisation socio-économique des États membres de I'UE ont conduit à réduire la circulation intra-communautaire en raison de l'accroissement des salaires dans les pays européens d'émigration. Ainsi, la valeur relative des travailleurs des pays tiers s'est accrue durant la période fordiste. Cependant, les années 2000 connaissent un retour puissant de ce mécanisme

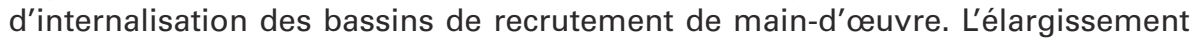
de I'UE va de pair avec le retour de l'internalisation au sein de I'UE des bassins de recrutement, ce qui permet de rester dans le modèle du travail temporaire, de permettre par le travail immigré un accroissement des richesses dans le pays d'origine d'autant plus qu'y sont aussi délocalisées des entreprises industrielles, et surtout limite la diversification des origines nationales des migrants suivant en cela le mode de politique d'immigration décrit par Gary P. Freeman (1995). La libre circulation des services et le détachement du personnel fournissent en cela une nouvelle opportunité à ce processus d'internalisation. C'est pourquoi l'étude du travail immigré suppose de porter une attention particulière aux ressortissants européens, et pas seulement à ceux provenant des pays tiers.

\section{Présence des étrangers sur le marché du travail}

Plusieurs recherches (Martens, 2004 ; Martens et Ouali, 2005) ont établi l'existence d'une stratification ethnique du marché du travail en Belgique. Ainsi, les travailleurs étrangers ou d'origine étrangère sont surreprésentés dans les secteurs du nettoyage industriel, des centres d'appels, de la logistique et du transport, de l'horticulture, de la construction, de la domesticité, des restaurants et hôtels. Malgré la faiblesse des échantillons, les enquêtes européennes sur les forces de travail donnent une représentation des trois indicateurs fondamentaux (taux d'emploi, taux de chômage et taux d'activité) pour comparer la population belge à la population étrangère sur le marché du travail belge. Comme le montre le tableau 1, le taux d'activité des Européens est légèrement plus élevé que celui des Belges, témoignant de leur forte participation au marché du travail. II donne aussi une indication du poids occupé par les travailleurs européens par rapport à ceux des pays tiers. Par ailleurs, le taux d'emploi des Belges est légèrement plus élevé que celui des Européens (UE-27), et surtout nettement plus élevé que celui des ressortissants de pays tiers. 
Tableau 1 : Taux d'activité, d'emploi et de chômage par nationalité et pays de naissance, en 2010

\begin{tabular}{|l|l|l|l|l|l|l|}
\cline { 2 - 7 } \multicolumn{1}{c|}{} & $\begin{array}{l}\text { Force de } \\
\text { travail } \\
\text { (de 15 à } \\
\text { 64 ans) }\end{array}$ & Employé & $\begin{array}{l}\text { Sans } \\
\text { travail }\end{array}$ & $\begin{array}{l}\text { Taux } \\
\text { d'activité }\end{array}$ & $\begin{array}{l}\text { Taux } \\
\text { d'emploi }\end{array}$ & $\begin{array}{l}\text { Taux de } \\
\text { chômage }\end{array}$ \\
\hline Belge & 6477957 & 4069876 & 331040 & $67,9 \%$ & $62,8 \%$ & $7,5 \%$ \\
\hline UE-27 & 471068 & 293634 & 36153 & $70,0 \%$ & $62,3 \%$ & $11,0 \%$ \\
\hline non UE & 227809 & 87079 & 38345 & $55,1 \%$ & $38,2 \%$ & $30,6 \%$ \\
\hline Total & 7176834 & 4450589 & 405538 & $67,7 \%$ & $62,0 \%$ & $8,4 \%$ \\
\hline
\end{tabular}

Source : Direction générale Statistique et Information économique -

Enquête sur les forces de travail.

En analysant spécifiquement le taux de chômage, les ressortissants des pays tiers (non UE) ont un taux presque trois fois plus important $(30,6 \%)$ que celui des Européens (11\%). Les Congolais, les Marocains et les Turcs ont des taux de chômage plus élevés que ceux des citoyens européens, respectivement de $37,6 \%, 37,3 \%$ et 30,2 \%. Les taux de chômage parmi les ressortissants européens connaissent une grande variation : de $20,2 \%$ et $19 \%$ pour deux anciennes migrations, les Italiens et les Grecs alors que ceux des Français $(9,5 \%)$ ou les Hollandais $(10,7 \%)$ sont plus proches de la moyenne. Cependant, les taux de chômage les plus bas s'observent parmi les citoyens des nouveaux pays adhérents. Le taux de chômage est particulièrement bas parmi les Polonais, les Roumains et les Bulgares, respectivement $5 \%, 5,5 \%$ et de 8,6 \%. En 2010, le taux de chômage des femmes étrangères des pays tiers est beaucoup plus grand $(34,5 \%)$ qu'il ne l'est pour les femmes belges $(7,7 \%)$ ainsi que pour les femmes européennes (UE-27) (11,4 \%). Par contre, le taux de chômage des personnes non-EU nées à l'étranger $(23,5 \%)$ est plus faible que celui des ressortissants des pays tiers $(30,6 \%)$. Enfin, il existe une grande disparité régionale liée à des situations économiques contrastées, meilleures en Flandre qu'à Bruxelles ou en Wallonie. Pour les ressortissants des pays tiers, le chômage est nettement plus bas en Flandre (24,7 \%) qu'en Wallonie (38,7\%) et à Bruxelles (32,3\%). De nombreux étrangers ont acquis la nationalité belge depuis 2000, à savoir plus de 400000 personnes (CECLR, 2012).

La présence des travailleurs étrangers sur le marché du travail peut être établie à partir de diverses bases de données relatives à la délivrance des permis de travail, au travail indépendant et au détachement du personnel. C'est ce que nous nous proposons d'analyser en cherchant à mettre en évidence comment le marché du travail en Belgique capte les ressortissants des nouveaux États membres et, tout particulièrement, les Polonais, les Roumains et les Bulgares. Depuis 1936, les permis de travail constituent le principal instrument public de régulation de la migration de travail en Belgique. Ainsi, tout étranger qui souhaite travailler en Belgique doit obtenir un permis de travail, sauf s'il en est dispensé. De même, l'employeur qui souhaite embaucher un étranger doit demander une autorisation d'occupation. Cet instrument a particulièrement été utilisé entre 1946 et 1974, période de la grande migration de travail en Belgique. 
Depuis cette époque, le nombre de demandes de permis de travail a fortement chuté sans pour autant disparaître. La législation relative aux permis de travail a fortement changé au cours des deux dernières décennies. Une importante refonte du système a été proposée avec la loi du 30 avril 1999 (Moniteur belge, 21/05/1999) qui a dû tenir compte des diverses catégories juridiques d'étrangers comprises dans la loi du 15 décembre 1980 (Moniteur belge, 15/02/2011) régissant l'accès au territoire et le séjour, et notamment en incluant le principe de la libre circulation des travailleurs ressortissants des États membres de I'Union européenne. Les citoyens de l'espace économique européen sont ainsi exemptés de l'obligation de la possession du permis de travail pour entrer sur le marché du travail. Cependant, à l'occasion de l'élargissement de l'Europe en 2004, la Belgique comme d'autres pays européens, a imposé des mesures transitoires à huit nouveaux États membres, notamment à la Pologne, obligeant ces citoyens européens à posséder un permis de travail pour entrer sur le marché du travail. Cette mesure transitoire a été levée le 1er mai 2009 alors que la Roumanie et la Bulgarie se la sont vu imposer à partir de 2007, et ce, au moins jusqu'en décembre 2013. Toutefois, ces ressortissants ont bénéficié de conditions spéciales. Ainsi, ils bénéficient de procédures accélérées de leurs demandes qui n'obligent pas l'agence de l'emploi à vérifier l'existence d'une réelle pénurie de main-d'œuvre sur le marché de l'emploi si le poste pour lequel le permis de travail est requis figure dans la liste des métiers en pénurie. Le permis $B$ a une durée maximale d'un an mais peut être renouvelé et est aussi utilisé pour le travail saisonnier. En outre, les étudiants étrangers et les demandeurs d'asile ont pu aussi bénéficier d'un permis de travail sur la base de la loi du 1er avril 2003. Ce permis d'une durée de douze mois et renouvelable est associé à la légalité de séjour.

Figure 1 : Permis de travail B (premiers et renouvellements) délivrés entre 2000 et 2010

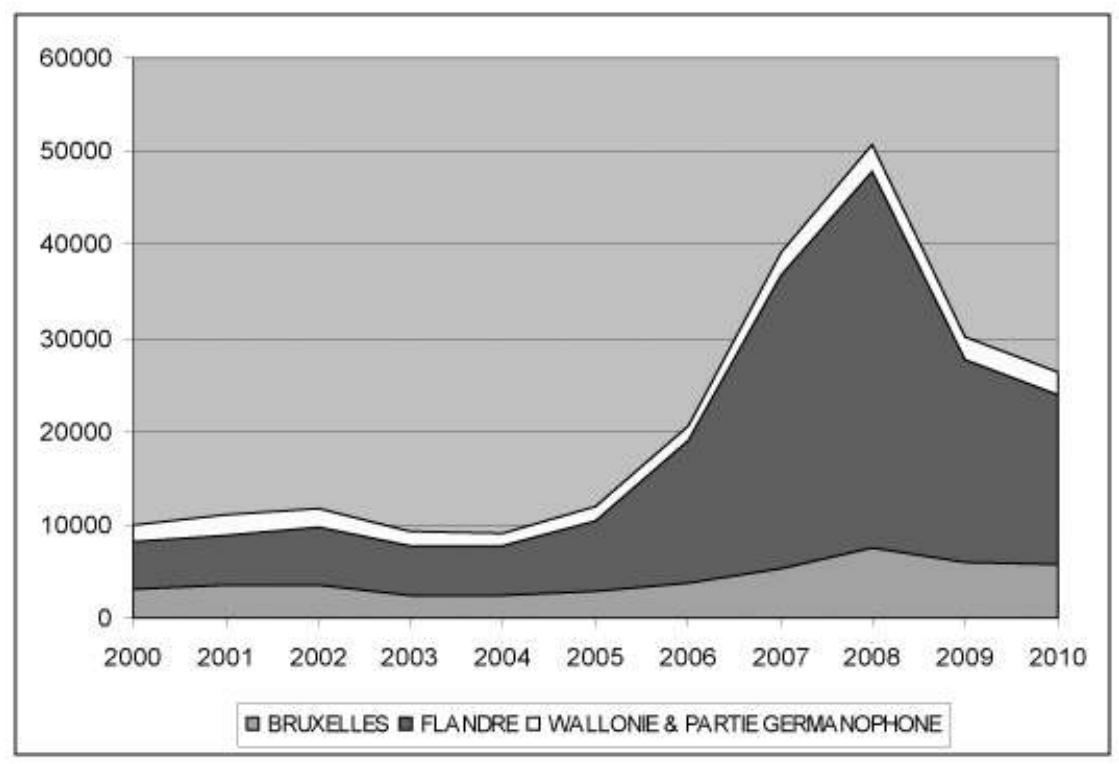

Source : Direction générale Statistique et Information économique. 
La figure 1 présente l'évolution des premiers permis de travail et ceux renouvelés entre 2000 et 2010 . Le nombre de permis de travail délivrés a augmenté à partir de 1995. Cette hausse dure jusqu'en 2001 à Bruxelles et en Wallonie, 2002 en Flandre. Après une légère baisse jusqu'en 2004, le nombre de permis $B$ octroyés repart à la hausse dans toutes les Régions à partir de 2005. Entre 2004 et 2008, le nombre de permis B a quintuplé (passant de 9105 à 50731 permis B). Cet accroissement continu est particulièrement marqué en Flandre. II s'explique par l'arrivée massive de travailleurs saisonniers des nouveaux États membres de I'UE employés dans I'horticulture. L'augmentation des permis B délivrés au cours de cette période tient principalement à son usage dans le secteur horticole flamand. Ce secteur à lui seul a attribué plus de la moitié des permis $B$ à partir de 2006 et à plus de $95 \%$ à des travailleurs polonais (Rosenfeld et al., 2010). La chute observée à partir de 2009 résulte d'un double processus. Le premier tient à la suppression des mesures transitoires pour les Polonais qui continuent à travailler dans le secteur de I'horticulture en n'ayant plus besoin de permis de travail. Le deuxième résulte du ralentissement économique durant l'année 2008. En 2008, le permis B est attribué pour 53,4 \% aux Polonais, $13,5 \%$ aux Roumains et $6,3 \%$ aux Bulgares. En 2010, les travailleurs roumains bénéficient de $39,2 \%$ des permis de travail octroyés et les Bulgares de $15 \%$. Ainsi, le permis de travail $B$, instrument historique de la régulation de la migration de travail, en vient à être utilisé majoritairement par les ressortissants des nouveaux États membres de I'UE durant les périodes de transition qui leur sont imposées.

Les permis de travail pour le personnel hautement qualifié concernent d'autres secteurs d'activités et surtout d'autres nationalités. De 2001 à 2010, le nombre de permis de travail $B$ délivrés pour les travailleurs hautement qualifiés est en forte croissance et a pratiquement doublé. Les professions concernées touchent l'ingénierie, l'information, le commerce, le management. Les travailleurs indiens sont les principaux bénéficiaires de ces permis de travail. Ils sont principalement occupés en Flandre bien que le nombre de permis délivrés par Bruxelles soit aussi très élevé. Les travailleurs hautement qualifiés proviennent principalement des pays tiers, et singulièrement de I'Inde, du Japon, des ÉtatsUnis et de la Chine (cf. Tableau 2), nationalités qui ont aussi connu une progression durant cette période. Inversement, les ressortissants des nouveaux États membres de I'UE sont pratiquement absents de cette catégorie de travailleurs.

Si la législation suppose que les salariés provenant des nouveaux États membres de l'UE soient soumis à l'obtention d'un permis de travail, il n'en va pas de même pour les travailleurs indépendants. II n'est donc pas étonnant de constater que l'augmentation des travailleurs indépendants étrangers soit fortement liée à l'élargissement de I'UE, en 2004 avec dix nouveaux États membres, et en 2007 avec l'arrivée de la Bulgarie et de la Roumanie. Selon, I'Institut national $d$ 'assurances sociales pour travailleurs indépendants, le nombre d'indépendants polonais avant 2004 est en forte croissance (938 en 2001, 1197 en 2003, 1732 en 2003 et 2803 en 2004) et est resté relativement stable jusqu'à une période récente. Le nombre de travailleurs indépendants bulgares et roumains a, quant à lui, augmenté depuis 2007. Le nombre de travailleurs indépendants a également augmenté pour d'autres nationalités européennes (France, Pays-Bas, Italie et Portugal). De même, le nombre de travailleurs indépendants des pays tiers a aussi crû durant les dernières années. Toutefois, l'étude particulière des indépendants polonais, roumains et bulgares peut laisser supposer que le 
Tableau 2 : Permis de travail B (premiers et renouvellements) pour les travailleurs hautement qualifiés, entre 2001 et 2010

\begin{tabular}{|l|l|l|l|l|l|l|}
\cline { 2 - 7 } \multicolumn{1}{c|}{} & $\mathbf{2 0 0 1}$ & $\mathbf{2 0 0 7}$ & $\mathbf{2 0 0 8}$ & $\mathbf{2 0 0 9}$ & $\mathbf{2 0 1 0}$ & $\begin{array}{l}\text { Différence } \\
\text { entre 2001 } \\
\text { et 2010 }\end{array}$ \\
\hline Inde & 508 & 1960 & 2581 & 2258 & 2469 & $+380,0 \%$ \\
\hline Japon & 588 & 1231 & 764 & 1116 & 1067 & $+81,5 \%$ \\
\hline USA & 795 & 904 & 716 & 1026 & 917 & $+15,3 \%$ \\
\hline Chine & 169 & 335 & 406 & 382 & 439 & $+160,0 \%$ \\
\hline Turquie & 138 & 375 & 429 & 370 & 354 & $+156,5 \%$ \\
\hline Canada & 205 & 207 & 185 & 240 & 214 & $+4,4 \%$ \\
\hline Russie & 167 & 243 & 215 & 238 & 195 & $+16,8 \%$ \\
\hline Brésil & - & - & 135 & 165 & 147 & $+147 \%$ \\
\hline Roumanie & 153 & 145 & 158 & 126 & 166 & $+8,5 \%$ \\
\hline Autres & 1774 & 2139 & 2136 & 1874 & 1916 & $+8,0 \%$ \\
\hline Total & 4497 & 7539 & 7725 & 7795 & 7884 & $+75,3 \%$ \\
\hline
\end{tabular}

Source : Service public fédéral Emploi, Travail et Concertation sociale.

Tableau 3 : L'emploi indépendant et aidant des indépendants ayant débuté leur activité durant l'année, de 2006 à 2010

\begin{tabular}{|l|l|l|l|l|l|}
\cline { 2 - 6 } \multicolumn{1}{c|}{} & $\mathbf{2 0 0 6}$ & $\mathbf{2 0 0 7}$ & $\mathbf{2 0 0 8}$ & $\mathbf{2 0 0 9}$ & $\mathbf{2 0 1 0}$ \\
\hline Roumanie & 1009 & 2640 & 2348 & 2620 & 3704 \\
\hline Pologne & 2411 & 2754 & 2094 & 1799 & 1793 \\
\hline Pays-Bas & 1752 & 1767 & 1631 & 1580 & 1692 \\
\hline Bulgarie & 209 & 1347 & 1540 & 1666 & 2367 \\
\hline France & 1269 & 1215 & 1364 & 1288 & 1410 \\
\hline Italie & 865 & 910 & 966 & 886 & 983 \\
\hline Portugal & 453 & 480 & 567 & 622 & 640 \\
\hline Maroc & 427 & 465 & 455 & 479 & 503 \\
\hline Turquie & 415 & 441 & 473 & 424 & 483 \\
\hline Autres & 2981 & 3071 & 3024 & 3315 & 3841 \\
\hline $\begin{array}{l}\text { Total des } \\
\text { étrangers }\end{array}$ & 11791 & 15090 & 14464 & 14679 & 17416 \\
\hline Belgique & 60082 & 65712 & 65285 & 60913 & 67616 \\
\hline Total & 71873 & 80802 & 79749 & 75592 & 85032 \\
\hline
\end{tabular}

Source : Institut national d'assurances sociales pour travailleurs indépendants. 
recours abondant au statut d'indépendant constitue un contournement de la législation relative sur le permis de travail ou sur l'emploi salarié et concerne au moins en partie un usage de faux indépendants, situation que nous avons retrouvée lors de plusieurs recherches (Martiniello et al., 2010 ;Timmerman et al., 2012). Ce système est notamment utilisé dans le secteur de la construction où des employeurs refusent d'engager comme salariés des travailleurs et leur suggèrent $d^{\prime}$ 'acquérir un statut d'indépendant.

Depuis la transposition de la directive sur les prestations de services (directive 96/71/CE) et sur la réglementation sur le détachement des travailleurs en 2002, le nombre de travailleurs détachés qui ne sont pas pris en charge par la sécurité sociale belge a fortement crû. La Belgique a institué un système d'enregistrement des détachements de personnel, la base de données LIMOSA, qui fournit en partie une indication de la délocalisation intérieure. Certes, tous les détachements de personnel ne relèvent pas de ce processus spécifique. Quand des entreprises multinationales font venir des travailleurs du siège central dans une filiale, le processus est différent. En ce sens, il serait faux de penser que le détachement du personnel soit réductible à un processus de délocalisation intérieure. Cependant, dans certains cas il l'est. Le ralentissement économique a réduit le nombre de travailleurs détachés en 2010 par rapport aux années antérieures. La plupart des entreprises qui utilisent le détachement du personnel ont leur siège social en Europe et sont situées dans les pays limitrophes. : Pays-Bas (20 683 personnes en 2009 et 11065 en 2010), I'Allemagne (15 347 personnes en 2009 et 7034 en 2010) et la France (12 242 personnes en 2009 et 5994 en 2010). Toutefois, il est important de constater que les trois nouveaux États membres utilisent aussi fortement le dispositif du détachement de personnel, en particulier la Pologne (12 225 personnes en 2009 et 6041 en 2010) et dans une moindre mesure, la Roumanie (2 804 personnes en 2009 et 1041 en 2010).

Tableau 4 : Évolution des détachements de personnels de 2007 à 2010, par nationalité

\begin{tabular}{|l|l|l|l|l|l|}
\cline { 2 - 6 } \multicolumn{1}{c|}{} & $\mathbf{2 0 0 7}$ & $\mathbf{2 0 0 8}$ & $\mathbf{2 0 0 9}$ & $\mathbf{2 0 1 0}$ & \% en 2010 \\
\hline $\begin{array}{l}\text { Total } \\
\text { UE-15 }\end{array}$ & 51988 & 66545 & 59957 & 30258 & $72,0 \%$ \\
\hline $\begin{array}{l}\text { Total } \\
\text { UE-10 }\end{array}$ & 11189 & 15857 & 14918 & 7262 & $17,3 \%$ \\
\hline Total UE-2 & 1234 & 3286 & 3386 & 1582 & $3,8 \%$ \\
\hline Non UE & 5926 & 8074 & 6162 & 2944 & $7,0 \%$ \\
\hline Total & 70337 & 93762 & 86432 & 42046 & $100 \%$ \\
\hline
\end{tabular}

Source : LIMOSA, DG Inspection sociale.

Les deux principaux secteurs utilisateurs de ce système sont la construction et l'industrie manufacturière, et dans une moindre mesure I'horticulture. Dans I'industrie manufacturière, les travailleurs polonais sont en quatrième position après les Français, les Allemands et les Hollandais. Par contre, dans le domaine de la construction, la Pologne est le premier fournisseur de personnel détaché. Ce seul secteur représente plus de la moitié des travailleurs détachés polonais en Belgique. Dans I'horticulture, les Polonais sont en deuxième position après 
les Hollandais. La construction et l'horticulture représentent $75 \%$ des travailleurs polonais détachés. La directive sur les services (Directive 96/71/CE) ne permet pas seulement des migrations intra-communautaires pour les citoyens européens. Elle offre aussi de nouvelles possibilités de migrer, au sein de I'UE, sans obligation de permis de travail, pour les ressortissants de pays tiers dans la mesure où ils ont un permis de séjour de plus de trois mois et sont employés légalement dans un État membre pendant la durée du travail à effectuer. Ils ne doivent pas disposer d'un permis de travail. Les ressortissants des pays tiers de I'UE représentaient $7 \%$ des travailleurs détachés (cf. Tableau 3). Majoritairement les travailleurs brésiliens sont détachés par des sociétés portugaises, les travailleurs marocains et algériens par des entreprises françaises et les travailleurs turcs par des firmes allemandes. L'usage accru de ce dispositif a également conduit les services d'inspection sociale à changer leur cible. En effet, durant les années 2000 les inspections sociales à Bruxelles avaient fait de la main-d'œuvre étrangère employée irrégulièrement la priorité de leur travail (Nagels et Rea, 2010). Depuis 2009, elles ont réorienté leur priorité sur ce dispositif.

Ce système constitue un dispositif représentant ce que certains acteurs européens voudraient promouvoir comme migration temporaire, déléguant même aux entreprises la charge de l'entrée et la sortie du territoire de ces travailleurs. L'accroissement du nombre de travailleurs immigrés, en particulier en provenance de Pologne, de Roumanie et de Bulgarie, donne à penser qu'il s'agit d'un nouvel instrument aussi de la politique de la migration de travail temporaire. Le dernier rapport de l'European Migration Network (2011) sur les migrations temporaires va dans ce sens en relevant que si la part des ressortissants de pays tiers est inférieure à $10 \%$, le nombre absolu de détachements de personnes de pays tiers est supérieur à celui des permis de travail pour la même population.

\section{L'insertion des nouveaux migrants dans des niches ethniques}

Les données statistiques présentées indiquent l'existence de concentrations de travailleurs dans des secteurs particuliers, notamment là où l'intensité de main-d'œuvre est forte. Pour mieux incarner ce processus, nous allons examiner la situation dans trois secteurs traditionnels : la construction, l'horticulture et la domesticité. La fragmentation du marché du travail permet aussi des formes de spécialisation dans la division du travail. Certaines activités professionnelles ou certains segments de secteur d'activité sont occupés par des travailleurs de même nationalité. Ceci conduit à la formation de niches ethniques. Ce concept proposé par Roger Waldinger (1994 et 1996) désigne la surreprésentation d'un groupe ethnique dans un secteur par rapport à sa part dans le volume total de l'emploi. Une des propriétés de la niche ethnique est de fonctionner comme une chaise musicale. Elle se construit sur la base de deux facteurs : d'une part les opportunités du marché du travail et celles de la mobilité internationale, et d'autre part les caractéristiques des groupes ethniques. Le principe de la chaise musicale autorise des changements d'occupation de places lorsque certaines deviennent vacantes. Des travailleurs immigrés quittent un poste pour laisser leur place à d'autres travailleurs, ce roulement pouvant répondre à une logique nationale et/ou ethnique. La construction des niches ethniques se réalise 
aussi à partir de la mobilisation de différents types de statuts dans l'emploi. Ceux-ci peuvent prendre des formes relativement classiques comme le permis de travail $B$ attribué au travailleur salarié, le permis de travail $C$ attribué aux demandeurs d'asile et aux étudiants étrangers résidant en Belgique, le travail indépendant ou le travail irrégulier. À ces formes s'ajoutent ou s'articulent de nouvelles formes de la dérégulation du marché de l'emploi comme le détachement de personnel.

L'usage de la sous-traitance et des firmes intérimaires dans la construction (Jounin, 2008) témoigne de ces nouvelles modalités de fonctionnement du marché de I'emploi qui constituent autant d'opportunités de recours à des travailleurs immigrés. Les données LIMOSA démontrent que dans le secteur de la construction, les travailleurs polonais sont très nombreux à être embauchés dans le cadre du détachement du personnel. Ainsi, le recours de la sous-traitance en cascade conduit à préférer établir des contrats avec des sociétés ayant leur siège en Pologne et venant effectuer les services pour lesquels elles sont payées avec leurs travailleurs plutôt que devoir recourir par exemple à des travailleurs polonais bénéficiant de permis de travail. L'usage de la réglementation sur la libre circulation des services vient, dans le secteur de la construction, renverser des pratiques antérieures de recrutement de travail. Ces sociétés agissent en particulier sur les grands chantiers du secteur de la construction et généralement pour les tâches les moins qualifiées.

Dans le sous-segment des travaux de rénovation des habitations privées ou des petites entreprises, un autre processus est à I'œuvre. Depuis 1991, de nombreux travailleurs polonais sont arrivés, en particulier à Bruxelles, pour travailler avant tout pour des particuliers. Ces travailleurs ont été très longtemps embauchés de manière irrégulière. Ils effectuent principalement des travaux de gros œuvre (maçonnerie), d'installation de chantier, de plafonnage et de pose de plaques de plâtres. Ils sont payés généralement $7 €$ à $8 €$ de I'heure et trouvent leur emploi par la mobilisation des réseaux sociaux. La demande de rénovation des habitations privées était très importante durant les années 1990 et 2000. N'étant pas soumis à l'obligation d'un visa, ils effectuent souvent des allers-retours entre la Podlasie, leur région d'origine et Bruxelles (Kuzma, 2012). Ils préfèrent en général les travaux dans les habitations pour éviter les contrôles de l'inspection sociale. Leur emploi est relativement instable parce qu'ils sont engagés pour des chantiers à durée limitée. Une fois ceux-ci terminés, ils se trouvent sans travail et doivent en chercher un autre. Dépourvus de droits sociaux, ils sont particulièrement précarisés et constituent la forme la plus dérégulée du travail. Au cours des années 1990, les travailleurs polonais constituaient les travailleurs sans-papiers les plus nombreux. Les différentes inspections sociales chargées de faire respecter les droits sociaux se sont alors concentrées de manière privilégiée sur ce contentieux particulier de l'occupation de main-d'œuvre étrangère (Nagels et Rea, 2010). De nombreux travailleurs interpellés par la police étaient alors rapatriés en Pologne. Avec l'entrée de la Pologne dans I'UE en 2004, la situation a changé. Certains travailleurs ont pu être engagés moyennant l'obtention d'un permis de travail alors que d'autres ont acquis le statut d'indépendant (vrai ou faux). Délaissant pour une part d'entre eux ce type d'emploi, ils ont été remplacés en partie par des Roumains. Cependant, le phénomène le plus visible a été la substitution des Polonais par les Brésiliens sur le même segment du marché du travail. 
L'arrivée des Brésiliens en Belgique trouve son origine principalement dans une opportunité d'accéder au territoire (Rosenfeld et al., 2009) sans être soumis à l'obligation de disposer d'un visa C (trois mois), un visa d'entrée sur le territoire (ou visa de tourisme) à l'inverse de nombreux pays de provenance des migrants latino ou africains. Ils constituent un exemple classique de l'overstayer. Après les trois mois de séjour légal, ils entrent dans la clandestinité. Les hommes brésiliens vont en partie prendre la place occupée par les travailleurs polonais dans la rénovation d'habitations privées et de petites entreprises. Ce sont des travailleurs irréguliers qui effectueront des travaux de finition tels que la peinture, le plafonnage et la pose de plaques de plâtres. Des segments du secteur de la construction étaient occupés durant un temps par des travailleurs portugais. Une partie d'entre eux se sont convertis en entrepreneurs et vont embaucher des travailleurs brésiliens. Ces entrepreneurs ne sont souvent que des sous-traitants de plus grosses sociétés. Ce schéma peut prendre des formes variables : soit le travailleur brésilien travaille de manière irrégulière sur le chantier du patron qui l'engage, soit il travaille sur un chantier dont le patron est un sous-traitant. Dans certaines situations, le travailleur ne sait même pas qui est son patron (Rosenfeld, Marcelle et Rea, 2010). Pour se procurer un travail, les Brésiliens dépendent largement d'un intermédiaire. Ces intermédiaires fonctionnent comme des gangmasters (Geddes, 2005) constituant les équipes de travail. Les conditions de travail et les salaires de ces travailleurs sont identiques à ceux de leurs prédécesseurs polonais.

L'horticulture belge a longtemps fonctionné sans avoir recours aux travailleurs saisonniers étrangers. En effet, les besoins de main-d'œuvre saisonnière étaient satisfaits par la population locale (femmes au foyer, retraités, étudiants). La défection de ces travailleurs saisonniers traditionnels et l'accroissement de la production conduisent le Boerenbond, puissante organisation catholique flamande coordonnant le réseau de guildes agricoles rurales, à faire pression auprès des autorités politiques pour élargir les modes de recrutement. Ces dernières introduisent alors deux réglementations nouvelles : I'autorisation provisoire de travail pour les demandeurs d'asile et la création du statut de travailleur saisonnier et occasionnel. Les premiers travailleurs étrangers affectés au travail saisonnier sont les demandeurs d'asile d'origine indienne installés dans la province du Limbourg. D'abord engagés de manière irrégulière par les exploitants, ils seront autorisés à partir de 1994 à travailler durant soixante-cinq jours par an dans l'horticulture. En 1999-2000, les sans-papiers concernés par la campagne de régularisation bénéficient également du dispositif d'autorisation provisoire de travail (Botterman, 2001). Selon une allocation des travailleurs devenue classique, le secteur embauche des travailleurs réguliers et stables en nombre réduit, des travailleurs saisonniers dont les demandeurs d'asile, et les " régularisables ", et enfin des travailleurs irréguliers. Ceci représente le principe de la sur-disponibilité de main-d'œuvre en période de récolte décrit par JeanPierre Berlan (1986) et que I'on retrouve dans de nombreux pays européens (Morice et Michalon, 2008). L'instauration d'un statut de " travailleur saisonnier et occasionnel ", soumis à un régime spécial en matière de charges sociales, permet aussi d'introduire une grande flexibilité dans l'embauche. Le contrat de travail n'est conclu que pour une seule journée et le travailleur reçoit une " carte de cueillette " sur laquelle sont indiqués les jours de travail. Cette carte lui permet de travailler soixante-cinq jours maximum par an. Les années 2000 voient de nombreux changements. En 2003, I'autorisation provisoire d'occu- 
pation pour demandeurs d'asile est remplacée par le "permis de travail C " attribué aux demandeurs d'asile et aux étudiants étrangers. Par ailleurs, face aux pénuries de main-d'œuvre, I'horticulture est reprise dans la liste des métiers en pénurie pour lesquels une autorisation de recours à la main-d'œuvre étrangère est autorisée. Le recours au permis $B$ devient alors un instrument important de recrutement de main-d'œuvre. Ceci explique l'accroissement des permis $\mathrm{B}$ délivrés en Flandre aux travailleurs saisonniers polonais à partir de 2004, travailleurs soumis aux mesures transitoires. L'augmentation considérable des permis B à partir de 2004 tient aussi à I'assouplissement de la procédure d'attribution des permis de travail pour les ressortissants de l'un des nouveaux États membres. Pour cette catégorie de travailleurs, la procédure d'octroi du permis se fait sans examen préalable du marché du travail et le délai d'attribution ne doit pas excéder cinq jours. Malgré ces assouplissements et peu avant la suppression des mesures transitoires, des exploitants vont avoir de plus en plus recours à une autre modalité d'occupation de la main-d'œuvre étrangère : le détachement du personnel. Ce dernier représente un exemple de la délocalisation intérieure qui, à la différence de la délocalisation sur place (Terray, 1999), concerne des travailleurs en situation régulière tant du point de vue du séjour que du travail. Cette pratique s'est généralisée tant en Flandre (Boels, 2012) qu'en Wallonie (Rosenfeld, Marcelle et Rea, 2010). Souvent d'anciens travailleurs ou des membres de la famille de travailleurs polonais ont constitué une société en Pologne et continuent de travailler pour le même exploitant, parfois avec les mêmes travailleurs, mais avec un autre statut. Les principales différences entre les travailleurs avec permis de travail et les détachés tiennent à une moins bonne protection sociale de ces derniers et à une externalisation des procédures administratives et des coûts du recrutement de la main-d'œuvre. Le détachement de personnel fait naître une nouvelle modalité dans le processus migratoire : le déplacement de travailleurs immigrés qui ne sont pas reconnus comme tels. Leur identité n'est plus attachée au pays dans lequel ils travaillent. Ils sont en quelque sorte "désencastrés " socialement puisqu'ils dépendent de leur entreprise. La nature du lien devient alors davantage économique et moins sociale et politique. Le référent est davantage le marché que l'État.

La domesticité est un secteur traditionnel des femmes migrantes. Les femmes domestiques ont souvent été une part invisible de la migration de travail (Morelli et Gubin, 2004). Après les femmes de ménage italienne, espagnole, portugaise, marocaine et turque apparaissent celles d'origine polonaise, brésilienne, philippine et latino. Dans ce secteur aussi s'observe cette imbrication particulière entre origine nationale et articulation des statuts d'emploi. La domesticité est une activité de déplacements internationaux des femmes (Kofman, 2010). Le recours à une femme de ménage repose sur le principe de la délégation à une travailleuse étrangère des tâches domestiques qui revenaient auparavant aux femmes au sein du couple ou de la famille. La migration d'une femme devenue domestique laisse dans son propre foyer une place vide. Par conséquent, les enfants restés au pays sont pris en charge par une autre femme employée par la mère migrante. Ce phénomène qualifié de Global Care Chain par Arlie Russell Hochschild (2000) consiste en une série de liens personnels établis à travers le monde autour des tâches (rémunérées ou non) relevant du care. Pour Rhacel Parreñas (2001), ces déplacements en chaîne se fondent sur une division internationale du travail de reproduction. Cette chaîne du care se développe là où la question de l'égalité homme/femme face aux tâches domestiques est résolue par 
l'emploi d'une autre femme et en Europe, souvent avec le soutien public (Morel, 2007). Ainsi, l'émancipation de certaines femmes belges peut-elle s'appuyer sur une main-d'œuvre féminine précaire et étrangère dont le déplacement provoque à son tour le recrutement d'autres femmes pour les activités telles que les soins aux personnes et le nettoyage domestique?

À partir de 1991, Bruxelles connaît un accroissement des femmes de ménage d'origine polonaise provenant principalement de la Podlasie, une région du nord-est de la Pologne. S'inscrivant dans un modèle de circulation migratoire (Morokvasic et Rudolph, 1996), de nombreuses femmes vont trouver un emploi auprès de familles des classes moyennes et supérieures belges ainsi que des familles de travailleurs internationaux, nombreux à Bruxelles (Commission et Parlement européens, OTAN). Ces femmes polonaises en situation irrégulière en matière de séjour sont embauchées au noir comme femmes de ménage principalement en live out. Une partie de ces femmes circulent entre leur village d'origine et Bruxelles, entrant et sortant tous les trois mois du pays (Kuzma, 2012). D'autres s'installent dans la clandestinité. Les femmes d'ouvrage polonaises sont très prisées. Le qualificatif de "polonaise " associé au travail de femme de ménage constitue en soi la qualification même de la travailleuse. "Polonaise" signifiant travailleuse, consciencieuse, autonome. La recherche d'un emploi se déroule via les réseaux sociaux et familiaux. Les journaux gratuits en français et en polonais constituent également des sources de recrutement de travailleuses. La norme salariale qui s'est imposée à cette époque était de $6 €$ à $7 €$ de l'heure. Lorsqu'une immigrée rentre en Pologne pour les vacances ou pour des raisons familiales, sa place est occupée par une sœur, une cousine ou une amie, ce qui lui permet de reprendre son emploi chez les mêmes employeurs à son retour. Cependant, l'organisation de ce marché informel repose aussi sur le rachat du poste de travail. Si une heure de ménage est payée $6 €$, le poste est vendu $25 €$. Travaillant depuis plusieurs années chez les mêmes employeurs, les femmes polonaises ont un emploi qui est plus stable que celui des hommes. Par ailleurs, elles sont, compte tenu du caractère privé de leur lieu de travail, plus à l'abri des contrôles policiers que les hommes qui travaillent sur des chantiers à l'extérieur. En outre, à l'inverse des hommes polonais travaillant souvent entre eux, les femmes peuvent, en raison de leurs contacts fréquents avec leurs employeuses belges, forger des liens faibles (Granovetter, 1973) que certaines ont notamment mobilisés pour régulariser leur statut de séjour lors de la campagne de régulation en 2000 (Marx et al.2008; Godin et Rea, 2010).

Ce marché informel de la domesticité va connaître un grand changement en 2004. Comme d'autres pays européens, la Belgique institue un système de services d'aide aux personnes appelé " titres-services ". Ce système vise d'une part, à régulariser le travail au noir de nombreuses femmes de ménage, et d'autre part à organiser un secteur pourvoyeur d'emplois au-delà de l'aideménagère (courses, transport de personnes à mobilité réduite, garde d'enfants, etc.). Le titre-service, unité de paiement, circule dans un système comportant quatre acteurs : I'organisme fédéral gestionnaire des échanges de titres-services (actuellement la société Sodexho), I'entreprise agréée " titres-services " (entreprises publiques, privées non-commerciales ou privées commerciales), la travailleuse et le client. Les titres-services sont achetés par le client pour des tâches précises et ce dernier bénéficie d'une déduction fiscale à leur achat. Une aide-ménagère embauchée dans un contrat de travail par une société agréée 
vient effectuer ces tâches au domicile du client. Ce dernier remet en échange des heures prestées les titres services correspondants. L'aide-ménagère restitue tous les titres-services à son employeur qui les envoie à l'organisme payeur qui à son tour rembourse l'employeur. Le tarif horaire payé par le client est pratiquement le même que celui demandé dans le cadre du travail informel (7,50 € de I'heure jusqu'en 2013). Jouant sur l'avantage fiscal et la possibilité de régulariser l'emploi de la travailleuse en lui ouvrant le bénéfice de la protection sociale, de nombreux clients sont passés du système informel aux titres-services. Ce système combine subvention publique pour les demandeurs et soutien de l'offre (Henry et al., 2009).

En 2010, le système employait 136915 travailleurs et travailleuses dont $75,5 \%$ sont Belges (Idea, 2011). Cependant, Bruxelles connaît une situation particulière. À l'inverse des deux autres régions, la majorité des travailleuses bruxelloises sont étrangères. Ainsi, sur les 17256 travailleuses, $75 \%$ sont étrangères (Idea, 2011). Les travailleuses polonaises y sont les plus nombreuses (7,5\% des travailleuses pour toute la Belgique), mais de nombreuses nouvelles nationalités apparaissent (Brésilienne, Philippine, Équatorienne, etc.). De très nombreuses travailleuses polonaises sont passées du marché informel au marché formel. Dans le même temps, l'accroissement de la demande a conduit aussi de nombreuses travailleuses polonaises à changer de statut. Ainsi, certaines ont constitué des sociétés agréées de titres-services et sont devenues des employeurs. Les nouvelles demandes et les offres laissées vacantes par des travailleuses polonaises qui ont quitté le secteur sont dorénavant remplies par de nouvelles femmes migrantes. Ainsi, le marché du travail domestique bruxellois longtemps dominé par les femmes polonaises commence à voir apparaître des travailleuses venant des Philippines et de l'Amérique latine (Équateur, Colombie et Brésil) (Freitas, Godin et Rea, 2010). Ces dernières nationalités constituent des candidates pour ce que Roger Waldinger (2000) nomme les lousy jobs.

\section{Conclusion}

Malgré un taux de chômage important, la migration de travail n'a pas disparu en Belgique. Elle a surtout changé de forme. Elle répond toujours à sa fonction initiale, à savoir affecter des travailleurs dans des emplois socialement dépréciés, mal payés et dépourvus d'une bonne protection sociale, en particulier aujourd'hui dans le secteur des services et de la construction. Les migrations de travail ne se réduisent pas au travail irrégulier des sans-papiers et au travail des migrants réguliers soumis à l'obligation de posséder un permis de travail. Ces deux catégories existent bien mais au sein des entreprises d'autres figures du travail migrant ont pris place. Comme nous l'avons montré, les travailleurs ressortissants des nouveaux États membres de I'UE doivent être considérés comme des travailleurs immigrés et non des travailleurs en mobilité. La Belgique comme d'autres pays de l'Europe du Nord ont internalisé au sein de l'UE leur bassin de recrutement de travailleurs affectés aux emplois sales et moins bien rémunérés. II importe de ne pas faire sortir de l'étude de la migration de travail la situation des nouveaux ressortissants des États membres de I'UE qui constituent en Belgique une bonne part de ces nouveaux travailleurs immigrés et sans doute correspondent à l'idéal typique de la migration temporaire et circulaire. Si la délocalisation sur place a souvent été appréhendée comme la forme dominante 
et contemporaine de la migration de travail, la dérégulation du marché du travail a conduit à une plus grande variabilité des formes de travail immigré. Les entreprises outre le recours aux sans-papiers et aux travailleurs migrants réguliers disposent de la possibilité d'utiliser des travailleurs en tant qu'indépendant mais surtout aujourd'hui de faire appel au détachement de personnel. L'usage de ce dernier instrument de la régulation du marché du travail est très important en volume puisque les travailleurs de trois pays de I'UE (Pologne, Roumanie, Bulgarie) et des pays tiers qui travaillent en Belgique avec ce statut sont plus nombreux que ceux ayant un permis de travail. Le dumping social que cet instrument offre contribue à en faire une forme de délocalisation intérieure légale à la différence de la délocalisation sur place. Les diverses formes du travail immigré reposent dorénavant sur l'articulation des formes dérégulées du marché du travail et la diversification des origines nationales, au sein de l'UE et hors de I'UE.

\section{Références bibliographiques}

Adam Ilke, Ben Mohamed Nadia, Kagné Bonaventure, Martiniello Marco et Rea Andrea (2002) Histoire sans-papiers, Bruxelles, Édition Vista, 227 p.

Ambrosini Maurizio (2005) Sociologia delle migrazioni, Bologna, II Mulino, 294 p.

Anderson Bridget (2000) Doing the Dirty Work? The Global Politics of Domestic Labour, New York, Zed Books, 213 p.

Berlan Jean-Pierre (1986) Agriculture et migrations, Revue Européenne des Migrations Internationales, 2 (3), pp. 9-31

Boels Dominique (2012) The Belgian informal economy: a case study of seasonal work in fruit-growing in South Limburg, Working paper, [online]. URL: http://hdl. handle.net/1854/LU-3032434

Botterman Chris (2001) Arbeidsaanbod in de land- en tuinbouw: dringende maatregelen gevraagd, Brief, Informatie van de Boerenbond.

Brun François (2003) Les immigrés et l'évolution du marché du travail en France, Migrations société, 15 (85), pp. 67-78.

Castles Stephen (2002) Migration and Community Formation under Conditions of Globalization, International Migration Review, 36 (4), pp. 1143-1168.

Catarino Christine et Mirjana Morokvasic (2005) Femmes, genre, migration et mobilités, Revue Européenne des Migrations Internationales, 21 (1), pp. 7-27.

CECLR (Centre pour l'égalité des chances et la lutte contre le racisme) (2012) Migrations. Rapport annuel, Bruxelles.

EMN (European Migration Network) (2011) Temporary and Circular Migration: empirical evidence, current policy practice and future options in EU Member States, EMN Synthesis Report.

Freeman Gary P. (1995) Modes of Immigration Politics in Liberal Democratic States, International Migration Review, 29 (4), pp. 881-902. 
Freitas Any, Godin Marie et Rea Andrea (2012) Genre et immigration LatinoAméricaine en Belgique, in Christiane Timmerman, Marco Martiniello, Andrea Rea et Johan Wets Eds., Femmes dans les processus migratoires contemporains, Gent, Academia Press, pp. 132-180.

Geddes Andrew (2005) Chronicle of a Crisis Foretold: The Politics of Irregular Migration, Human Trafficking and People Smuggling in the UK, British Journal of Politics and International Relations, 7, pp. 324-339.

Godin Marie et Rea Andrea (2010) Une analyse genrée de la population de sanspapiers régularisés lors la campagne de 2000 en Belgique, Migrations société, 22 (129-130), pp. 75-90.

Granovetter Mark (1973) The Strength of Weak Ties, American Journal of Sociology, 78 (6), pp. 1360-1380.

Guerry Linda (2007) Morelli Anne et Gubin Éliane (textes réunis et coordonnés par) (2004) Pour une histoire européenne des femmes migrantes. Sources et méthodes, Sextant, revue du Groupe interdisciplinaire d'Études sur les Femmes, Université libre de Bruxelles, 21-22, 2004, 302 p., CLIO. Histoire, femmes et sociétés, 26, [en ligne], consulté le 03 mai 2013. URL : http://clio.revues.org/7043

Henry Arnaud, Nassaut Stéphane, Defourny Jacques et Nyssens Marthe (2009) Economie Plurielle et régulation publique : le quasi-marché des titres-services en Belgique, Gent, Academia Press, 320 p.

Hochschild Arlie Russell (2000) Global Care Chains and Emotional Surplus Valu, in Anthony Giddens and Will Hutton Eds., On the Edge: Living with Global Capitalism, London, Jonathan Cape, pp. 132-133.

Hollifield James F. (1997) L'Immigration et l'État nation à la recherche d'un modèle national, Paris, L'Harmattan, $112 \mathrm{p}$.

Idea Consult (2011) Évaluation du régime des Titres-services pour les Services et Emplois de proximité. Rapport Annuel 2010, Bruxelles, SPF Emploi, Travail et Concertation Sociale, [en ligne]. URL : www.emploi.belgique.be/publicationDefault.aspx?id=26752\#AutoAncher11

Jounin Nicolas (2008) Chantier interdit au public, Paris, La Découverte, 274 p.

Kofman Eleonore (2010) Gendered Migrations and the Globalisation of Social Reproduction and Care: New Dialogues and Direction, in Marlou Schrover and Eileen Jane Yeo Eds., Gender, Migration and the Public Sphere 1850-2005, New York, Routledge, pp. 118-139.

Kuźma Elżbieta (2012) Émergence d'une communauté transnationale dans I'espace migratoire européen. Analyse de la migration polonaise à Bruxelles (2002-2009), Thèse de doctorat, Université libre de Bruxelles.

Lutz Helma (Ed.) (2008) Migration and Domestic Work: A European Perspective on a Global Theme, Aldershot, Ashgate, 212 p.

Martens Albert (2004) Discriminations et actions positives dans I'emploi en Flandre et à Bruxelles. Quelques résultats de recherche empiriques, in Marco Martiniello et Andrea Rea Éds., Affirmative action : des discours, des politiques et des pratiques en débat, Louvain-la-Neuve, Academia, pp. 161-195.

Martens Albert et Ouali Nouria (2005) Discriminations des étrangers et des personnes d'origine étrangère sur le marché du travail de la Région de BruxellesCapitale, Rapport de synthèse, Bruxelles, Actiris, $96 \mathrm{p}$. 
Martiniello Marco, Rea Andrea, Timmerman Christiane et Wets Johan (Éds.) (2010) Nouvelles migrations et nouveaux migrants en Belgique, Gent, Academia Press, $339 \mathrm{p}$.

Marx Ive, Rea Andrea, Verbist Gerlinde, Godin Marie et Corluy Vincent (2008) L'intégration sociale et économique des personnes ayant bénéficié de la procédure de régularisation en 2000 (loi de 1999), Bruxelles, Centre pour l'égalité des chances et la lutte contre le racisme, 175 p., [en ligne]. URL : http://germe.ulb. ac.be/uploads/pdf/articles\%20online/anciens/BeforeAfter-rapport.pdf

Morel Nathalie (2007) From Subsidiarity to "Free Choice": Child and Elder care Policy Reforms in France, Belgium, Germany and the Netherlands, Social Policy \& Administration, 41 (6), pp. 618-637.

Morice Alain (2006) Pas de séjour sans travail ou les pièges du contrat saisonnier, Migrations société, 18 (107), pp. 211-231.

Morice Alain et Michalon Bénédicte (2008) Les migrants dans l'agriculture : vers une crise de main-d'œuvre ?, Études rurales, 182, pp. 9-28.

Morokvasic-Muller Mirjana et Rudolph Hedwig (Éds.) (1996) Migrants. Les nouvelles mobilités en Europe, Paris, L'Harmattan, 288 p.

Nagels Carla et Rea Andrea (2010) De la criminalisation des travailleurs sanspapiers, Champ Pénal, 7, [en ligne]. URL : http://champpenal.revues.org/7865

Parreñas Rhacel (2001) Servants of Globalization: Women, Migration and Domestic Work, Stanford, Stanford University Press, 309 p.

Piore Michael J. (1979) Birds of Passage: Migrant Labor and Industrial Societies, Cambridge, Cambridge University Press, 240 p.

Rea Andrea (2010) Les transformations des régimes de migration de travail en Europe, in Alain Morice et Swanie Potot Éds., De l'ouvrier immigré au travailleur sans papiers. Les étrangers dans la modernisation du salariat, Paris, Karthala, pp. 307-315.

ReaAndrea (2006) L'ímmigration sans immigrés, in Stéphane Beaud, Joseph Confavreux et Jade Lindgaard Éds., La France invisible, Paris, La Découverte, pp. 439-442.

Rosenfeld Martin, Gois Pedro, Rea Andrea, Lenz Annika et Reyntjens Pascal (2009) Immigration brésilienne en Europe. Dimension transnationale, Hommes et Migrations, 1281, pp. 54-63.

Rosenfeld Martin, Marcelle Hélène et Rea Andrea (2010) Opportunités du marché de l'emploi et carrières migratoires. Étude de cas, in Marco Martiniello, Andrea Rea, Christiane Timmerman et Johan Wets Éds., Nouvelles Migrations et Nouveaux Migrants en Belgique, Gent, Academia Press, pp. 119-175.

Rosenfeld Martin, Rea Andrea, Herman Barbara et Jacobs Dirk (2010) Les permis de travail en Belgique, in Marco Martiniello, Andrea Rea, Christiane Timmerman et Johan Wets Eds., Nouvelles migrations et nouveaux migrants en Belgique, Gent, Academia Press, pp. 85-115.

Supiot Alain (1999) Au-delà de l'emploi. Transformations du travail et devenir du droit du travail en Europe, Paris, Flammarion, 321 p.

Terray Emmanuel (1999) Le travail des étrangers en situation irrégulière ou la délocalisation sur place, in Étienne Balibar, Monique Chemillier-Gendreau, Jacqueline Costa-Lascoux et Emmanuel Terray Éds., Sans-papiers : I'archaïsme fatal, Paris, La Découverte, pp. 9-34. 
Timmerman Christiane, Martiniello Marco, Rea Andrea et Wets Johan (Éds.) (2012) Femmes dans les processus migratoires contemporains, Gent, Academia Press, $314 \mathrm{p}$.

Waldinger Roger (2000) Who gets the lousy jobs?, in Peter Kivisto and Georganne Rundblad Eds., Multiculturalism in the United States: Current Issues, Contemporary Voices, New York, Sage Publications, pp. 134-173.

Waldinger Roger (1996) Still the Promised City?, Cambridge, Harvard University Press, $374 \mathrm{p}$.

Waldinger Roger (1994) The Making of an Immigrant Niche, International Migration Review, The Center for Migration Studies of New York, 28 (1), pp. 3-33. 


\section{Andrea Rea \\ Les nouvelles figures du travailleur immigré : fragmentation
des statuts d'emploi et européanisation des migrations}

Bien que la rhétorique politique insiste depuis plus de quarante ans sur la fin de l'immigration de travail, la Belgique comme de nombreux autres pays européens connaît un accroissement de travailleurs migrants. Cet afflux de travailleurs étrangers résulte principalement de demandes formulées par des segments du marché du travail en quête de travailleurs acceptant des emplois relevant des 3D-Jobs (Dangerous, Demanding and Dirty). Ces demandes sont satisfaites par trois processus migratoires contemporains : l'élargissement de I'UE aux pays de l'Est, I'accroissement des demandeurs d'asile ayant obtenu le statut de réfugié et la régularisation des sans-papiers. L'augmentation de travailleurs étrangers se caractérise aussi par une féminisation de cette nouvelle main-d'œuvre et par une diversification des statuts de travail. L'article soutient que les élargissements de l'UE ont permis une internationalisation du bassin de recrutement de la main-d'œuvre immigrée. Par ailleurs, la libre circulation des services constitue un nouvel instrument de la migration de travail s'ajoutant à l'utilisation de la politique des permis de travail, faisant du détachement du personnel une " délocalisation intérieure ».

\section{$\cdots$ New Figures of Migrant Workers: Fragmentation of Work Status and Europeanization of Migration}

Although political discourses have been announcing the end of work migration since more than 40 years, the number of migrant workers increases in Belgium like in other European countries. The flow of foreigner workers mainly results from those labour market sectors demanding workers who accept to take a job in the 3D-Jobs sector (Dangerous, Demanding and Dirty). Three contemporary migratory processes satisfy such demand: Enlarging Europe to Eastern European countries; increasing numbers of asylum seekers who obtain the refugee status; regularizing undocumented people. The increase of foreign workers is also characterized by the feminization of this new workforce and by the diversification of work statuses. The paper argues that the EU Enlargement has allowed the making of an internal space of migrant workers recruitment. Since the free movement of services is a new tool of work migration policy that is coupled with the use of work permits policy, the posted workers could be seen as an "internal outsourcing".

\section{Nuevas figuras de trabajadores migrantes: fragmentación del estatus laboral y la europeización de la migración}

A pesar de la retórica política ha anunciado más de 40 años al final de la migración laboral, el número de trabajadores migrantes aumenta en Bélgica, al igual que en otros países europeos. El flujo de trabajadores migrantes se compone principalmente por la demanda hecha por los sectores de trabajadores que acepten un puesto de trabajo en el 3D-Jobs (Dangerous, Demanding and Dirty). Estas demandas están satisfechas por tres procesos migratorios contemporáneos: la ampliación de la UE a los países del Este de Europa, el aumento del número de solicitantes de asilo que hayan obtenido la condición de refugiado, y la regularización de los sin papeles. El aumento de los trabajadores extranjeros también se caracteriza por una feminización de esta nueva fuerza de trabajo y una diversificación del estatus de trabajo. El papel sostiene que la ampliación de la UE ha permitido la creación de un espacio interno de la contratación para los trabajadores migrantes. Incluso la libre circulación de servicios es una nueva herramienta de las políticas migratorias de los trabajos que junto con el uso de la política de permisos de trabajo permite ver el desplazamiento de trabajadores como una "externalización interna". 